\title{
Acceptance of the Deltoid Muscle Injection of Aripiprazole Long-acting Injectable in the Patients with Schizophrenia
}

\author{
Hiroyuki Kamei ${ }^{1,2}$, Yuki Homma ${ }^{1}$, Ippei Takeuchi ${ }^{3}$, Genta Hajitsu ${ }^{4}$, Kaori Tozawa ${ }^{5}$, Masakazu Hatano ${ }^{1,2,6}$, \\ Aiko Fukui ${ }^{1}$, Manako Hanya ${ }^{1}$, Shigeki Yamada ${ }^{6}$, Nakao Iwata $^{2}$ \\ ${ }^{1}$ Office of Clinical Pharmacy Practice and Health Care Management, Faculty of Pharmacy, Meijo University, Nagoya, Aichi, ${ }^{2}$ Department of \\ Psychiatry, Fujita Health University School of Medicine, Toyoake, Aichi, ${ }^{3}$ Department of Psychiatry, Okehazama Hospital, Toyoake, Aichi, \\ ${ }^{4}$ Department of Psychiatry, Kamibayashi Memorial Hospital, Ichinomiya, Aichi, ${ }^{5}$ Department of Psychiatry, Kyowa Hospital, Obu, Aichi, \\ ${ }^{6}$ Department of Clinical Pharmacy, Fujita Health University School of Medicine, Toyoake, Aichi, Japan
}

\begin{abstract}
Objective: To improve poor medication adherence in schizophrenic patients, long-acting injectable (LAI) antipsychotics are used. However, it has not yet become common in Japan. Recently, aripiprazole LAl was approved for alternative injection into the deltoid muscle in addition to the gluteal muscle. The acceptance for the proposal to switch from gluteal to deltoid injections of aripiprazole LAI was investigated.

Methods: The subjects were 32 outpatients with schizophrenia who had continuously received aripiprazole LAI administration into the gluteal muscle for $\geq 6$ months. In the patients who had continued deltoid injection for 3 months after switching, the changes in the pain and shame in comparison with gluteal injections were evaluated.

Results: Switching to the deltoid injection was chosen by 17 out of 32 patients. Three months later, 9 patients were still receiving deltoid injections with highly rated satisfaction. The main reasons for switching to deltoid injections included the pain and shame associated with gluteal injections. The main reason for returning to the gluteal injection was the pain experienced from the injection in the deltoid.

Conclusion: The option to select the injected area was based on the amount of pain in the deltoid and gluteal sites, leading to the widespread use of aripiprazole LAI.
\end{abstract}

KEY WORDS: Aripiprazole; Antipsychotic; Schizophrenia; Long-acting injection; Gluteal; Deltoid.

\section{INTRODUCTION}

Schizophrenia is a chronic mental disorder characterized by positive, negative and cognitive symptoms. To treat schizophrenia, therapy with antipsychotics is primarily used. However, the discontinuation of drug therapy, often due to poor adherence, increases the risk of relapse after psychotic episodes [1]. This disorder recurred within 2 years in $\geq 50 \%$ of patients who had received acute treatment, and the recurrence rate in patients in whom drug therapy was discontinued was 5 times higher

Received: June 3, 2019/ Revised: August 2, 2019

Accepted: August 31, 2019

Address for correspondence: Hiroyuki Kamei Office of Clinical Pharmacy Practice and Health Care Management, Faculty of Pharmacy, Meijo University, 150

Yagotoyama, Tenpaku, Nagoya, Aichi 468-8503, Japan

E-mail: hkamei@meijo-u.ac.jp

ORCID: https://orcid.org/0000-0003-3778-2352 than in those continuing drug therapy [2]. Factors that influence medication adherence vary: the severity of psychiatric symptoms, lack of insight, polypharmacy, adverse-reaction-related reduction of the quality of life, and insufficient family support [3]. Ensuring treatment continuation through maintain/improve medication adherence is therefore a major objective in the treatment of schizophrenia.

As a strategy to overcome poor adherence in patients with schizophrenia, the use of long-acting injectable (LAI) of second-generation antipsychotics (SGA) is recommended [4]. As LAI is administered on outpatient visits, problems, such as daily-medication-related stress and forgetting to take medicine, may be overcome, and LAI may be useful for maintenance treatment for patients with poor medication adherence [5]. A study reported that the 2-year treatment continuation rate in patients receiving oral pre-

(ㄷ) This is an Open-Access article distributed under the terms of the Creative Commons Attribution Non-Commercial License (http://creativecommons.org/licenses/by-nc/4.0) which permits unrestricted non-commercial use, distribution, and reproduction in any medium, provided the original work is properly cited. 
parations was $63 \%$, whereas it was $82 \%$ in those receiving LAI [6]. Therefore, LAl should be recommended to patients with poor adherence and those aiming at rehabilitation. On the other hand, in Japan, the use of LAI has not been promoted. As one of the reasons, previously, firstgeneration antipsychotic LAI was primarily used. At that time, LAI was compulsively used to treat patients refusing drug therapy due to a lack of insight, but not to maintain/improve medication adherence [7]. Based on such a background, psychiatrists' speculation that patients/their families may not accept LAI and patients' anxiety/fears about injection have influenced the use of LAI despite the appearance of SGA-LAI on the market [8].

To improve poor medication adherence in patients with schizophrenia, SGA-LAI are often used in the USA and Europe. However, it has not yet become common in Japan. Currently, three types of SGA-LAI products are available in Japan: risperidone $\mathrm{LAI}$ (to be administered every two weeks), which became commercially available in 2009 as the first SGA-LAI in Japan, pariperidone (active metabolite of risperidone) LAI, and aripiprazole LAI, which acts, as a partial agonist, on dopamine $\mathrm{D}_{2}$ receptors. aripiprazole LAI, which was investigated in this study, is a preparation to be administered every 4 weeks $[9,10]$. Initially, the site of aripiprazole LAI administration was limited to the gluteal muscle, but administration into the deltoid muscle was also approved in March 2016 $[11,12]$. Pariperidone $L A I$ is available in Japan as same as Europe and USA for both gluteal or deltoid muscle injection [13], although the deltoid administration of risperidone LAI is not available in Japan. According to a previous study regarding acceptance for risperidone LAI administration into the deltoid muscle [14], 57\% of patients responding to a questionnaire wished to switch to deltoid muscle administration. A survey after 3 months showed that deltoid muscle administration had been continued in $60 \%$ of patients in the switching group, whereas it was again switched to gluteal muscle administration in $40 \%$, suggesting that resistance to the administration site influences LAI acceptance.

Thus, the administration of aripiprazole LAI into the deltoid muscle was newly approved, facilitating the selection of an administration site. Aripiprazole LAI therapy may be applied for a larger number of patients in Japan. In this study, we therefore investigated patients' acceptance and benefits for aripiprazole LAI administration into the deltoid muscle.

\section{METHODS}

\section{Study Design}

The subjects were 32 outpatients with schizophrenia who had continuously received aripiprazole LAI administration into the gluteal muscle for $\geq 6$ months in Fujita Health University Hospital, Okehazama Hospital, Kamibayashi Memorial Hospital, or Kyowa Hospital between July 2016 and June 2017. The patients aged 18 to 80 years with a diagnosis of schizophrenia based on the Diagnostic and Statistical Manual of Mental Disorders 4th edition, text revision criteria were eligible for study inclusion. Exclusion criteria were pregnancy, breast feeding, history of suicide attempt or psychiatric hospitalization during the past six months. After explaining this study to the subjects, written informed consent was obtained. This study was approved by the institutional review board of Fujita Health University (approval number: HM17-073).

After physicians explained the contents of this study to the patients, a questionnaire survey was conducted based on their consent. Initially, pharmacists explained the characteristics of deltoid/gluteal muscle aripiprazole LAI administration to the subjects, and provided necessary information. Then, Survey 1 , as described below, was conducted. Subsequently, gluteal muscle administration was switched to deltoid muscle administration in patients who wished to switch the administration site to the deltoid muscle. In those who had continued deltoid muscle administration for 3 months, Survey 2 was performed. In those in whom gluteal muscle administration was again introduced within 3 months after switching to deltoid muscle administration, Survey 3 was carried out (Fig. 1). Furthermore, the study design and questionnaire items were established based on a previous study [14], regarding acceptance for risperidone LAI administration into the deltoid muscle.

\section{Survey 1: Questionnaire survey regarding choices for an aripiprazole LAl administration site}

A questionnaire survey regarding choices for an aripiprazole LAI administration site and their reasons were conducted by inquiry. 


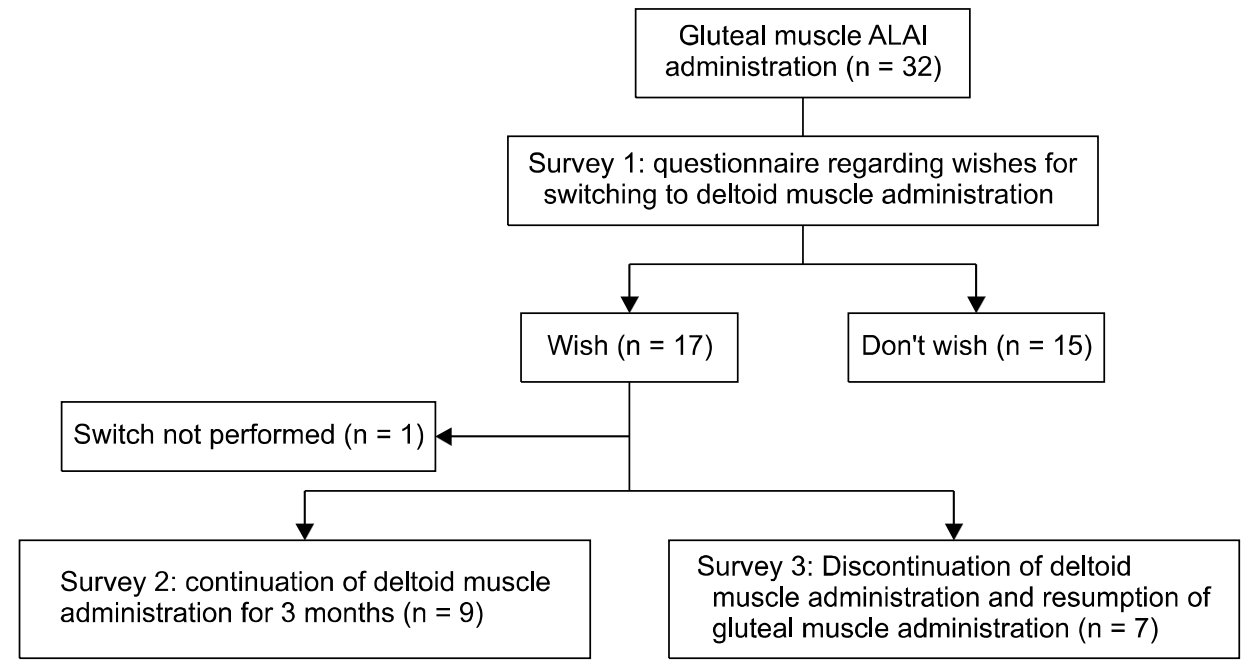

Fig. 1. Flow chart of the subjects. ALAI, Aripiprazole long-acting injection.

\section{Survey 2: Questionnaire survey regarding continuation}

This survey was conducted involving patients who had continued deltoid muscle administration for 3 months after switching. The changes in "shame", "pain”, "anxiety/fears", and "degree of satisfaction" in comparison with gluteal muscle administration were evaluated using 7 grades $(7$ : relatively present, 6 : present, 5 : slightly present, $4:$ no change, 3 : relatively absent, 2 : absent, 1 : nothing).

\section{Survey 3: Questionnaire survey regarding discontinuation}

This survey was conducted involving patients in whom deltoid muscle administration was discontinued within 3 months after switching to it, and gluteal muscle administration was again introduced. The reasons why they wished to discontinue deltoid muscle administration were investigated by inquiry.

\section{Evaluation of psychiatric symptoms}

The severity of psychiatric symptoms was evaluated using the Clinical Global Impressions severity ratings (CGI-S) [15]. It was assessed by attending physicians on consultation at the time of each questionnaire survey.

\section{Statistical Analysis}

For statistical analysis, we used IBM SPSS ver. 22 (IBM Co., Armonk, NY, USA). To compare patients who wished for deltoid muscle aripiprazole LAI administration with those who did not wish for it and the continuation group with the discontinuation group as the patient background, Student's $t$ test, Mann-Whitney $U$ test, chi square test and Fisher's exact test were used. Furthermore, changes in psychiatric symptoms in the continuation and discontinuation groups were analyzed using Wilcoxon's coded rank sum test. The $p$ values were two-sided except Fisher's exact test, and those less than 0.05 were considered significant.

\section{RESULTS}

\section{Patient Characteristics}

The mean age of the 32 subjects was $45.7 \pm 16.4$ years. They consisted of 12 males (37.5\%) and 20 females $(62.5 \%)$. The mean duration of disease was $14.3 \pm 10.6$ years. The mean body mass index (BMI) was $26.0 \pm 7.4$. The mean CGI-S score, which is a scale for rating psychiatric symptoms, was $4.2 \pm 1.5$.

\section{Survey 1: Questionnaire regarding choices for an aripiprazole $\mathrm{LAI}$ administration site}

Of the 32 subjects, 17 (53.1\%) wished to switch gluteal muscle administration to deltoid muscle administration, whereas 15 (46.9\%) did not wish it (Fig. 1).

No significant difference was found in the mean values of age, gender, duration of illness, BMl and CGI-S, and in the contents for co-medication, comorbidity, and physical diseases between those who wish to switch from gluteal to deltoid administration group and those who don't wish to switch from gluteal to deltoid administration group (Table 1).

The reasons for wishing for deltoid muscle administration included "pain on gluteal muscle administration" in 12 patients, "shame" in 7, "no necessity of lying on a 
bed for administration" in 7, and "comfortableness at the shoulder" in 2 (Fig. 2). The other reasons were "fears about 3 sessions of needle insertion on previous gluteal muscle administration" and "experience regarding intramuscular injection at the shoulder".

Table 1. Patient disposition in those who wish to switch from gluteal to deltoid administration group and those who don't wish to switch from gluteal to deltoid administration group

\begin{tabular}{|c|c|c|c|}
\hline Variable & $\begin{array}{l}\text { Wish to } \\
\text { switch from } \\
\text { gluteal to } \\
\text { deltoid } \\
(\mathrm{n}=17)\end{array}$ & $\begin{array}{l}\text { Don't wish to } \\
\text { switch from } \\
\text { gluteal to } \\
\text { deltoid } \\
(n=15)\end{array}$ & $p$ value \\
\hline Age (yr) & $43.7 \pm 18.0$ & $47.3 \pm 14.1$ & $0.523^{\mathrm{a}}$ \\
\hline \multicolumn{4}{|l|}{ Gender distribution (\%) } \\
\hline Female & 70.6 & 53.3 & \\
\hline Male & 29.4 & 46.7 & $0.314^{b}$ \\
\hline Duration of illness (yr) & $4.6 \pm 1.7$ & $3.7 \pm 1.1$ & $0.153^{c}$ \\
\hline Body mass index $\left(\mathrm{kg} / \mathrm{m}^{2}\right)$ & $26.2 \pm 6.8$ & $25.8 \pm 6.4$ & $0.878^{\mathrm{a}}$ \\
\hline CGI-S & $4.6 \pm 1.7$ & $3.7 \pm 1.1$ & $0.153^{c}$ \\
\hline Co-medication & 11 & 9 & $0.305^{\mathrm{b}}$ \\
\hline Antipsychotics & 4 & 4 & $0.579^{d}$ \\
\hline Antidepressants & 2 & 1 & $0.548^{\mathrm{d}}$ \\
\hline Benzodiazepines & 8 & 4 & $0.234^{b}$ \\
\hline Comorbidity & 1 & 2 & $0.452^{\mathrm{d}}$ \\
\hline Depression & 1 & 1 & $0.726^{\mathrm{d}}$ \\
\hline Dissociative disorder & 0 & 1 & $0.469^{d}$ \\
\hline Physical diseases & 5 & 2 & $0.254^{d}$ \\
\hline Hypertension & 1 & 2 & $0.452^{\mathrm{d}}$ \\
\hline Diabetes & 1 & 0 & $0.531^{\mathrm{d}}$ \\
\hline Hyperlipidemia & 1 & 0 & $0.531^{\mathrm{d}}$ \\
\hline Asthma & 1 & 0 & $0.531^{\mathrm{d}}$ \\
\hline Prostatomegaly & 1 & 0 & $0.531^{\mathrm{d}}$ \\
\hline Pollakiuria & 1 & 0 & $0.531^{\mathrm{d}}$ \\
\hline
\end{tabular}

Values are presented as mean \pm standard deviation or number only. CGI-S, Clinical Global Impressions-severity.

${ }^{\mathrm{a}}$ Student's $t$ test, ${ }^{\mathrm{b}}$ chi square test, 'Mann - Whitney $U$ test, ${ }^{\mathrm{d}}$ Fisher's exact test.
The reasons why the 15 patients did not wish for deltoid muscle administration included "pain on gluteal muscle administration" in 7, "no trouble for the current administration site (gluteal muscle)" in 4, "anxiety on seeing an injection needle at the time of administration" in 2, "experience regarding deltoid muscle injection" in 2, and "trace/hardening of the shoulder" in 1 (Fig. 3). The other reasons were "troubles on raising the arm", "necessity of taking clothes off", and "mild pain at the hip".

\section{Survey 2: Questionnaire regarding continuation}

Of the 17 patients who wished for deltoid muscle administration, it was continued for 3 months in 9 (52.9\%). Against initial decision pro switching one patient had not undergone the switch and therefore was not included in the follow-up assessment. To the question "How about your shame in comparison with the previous administration site (gluteal muscle)?", 5 patients selected "no shame", 2 selected "not shameful", 1 selected "not very shameful", and 1 selected "no change in comparison with the previous administration site". No patient selected "relatively shameful", "shameful", or "slightly shameful" (Fig. 4A). To the question "How about pain in comparison with the previous administration site (gluteal muscle)?”, 4 patients selected "not painful", 4 selected "not very painful", and 1 selected "no change". No patient selected "no pain”, "relatively painful”, "painful”, or "slightly painful" (Fig. 4B). To the question "How about anxiety/fears in comparison with the previous administration site (gluteal muscle)?", 2 patients selected "no anxiety/fear", 4 selected "not anxious/fearful", 2 selected "not very anxious/fearful", and 1 selected "no change". No patient selected "relatively

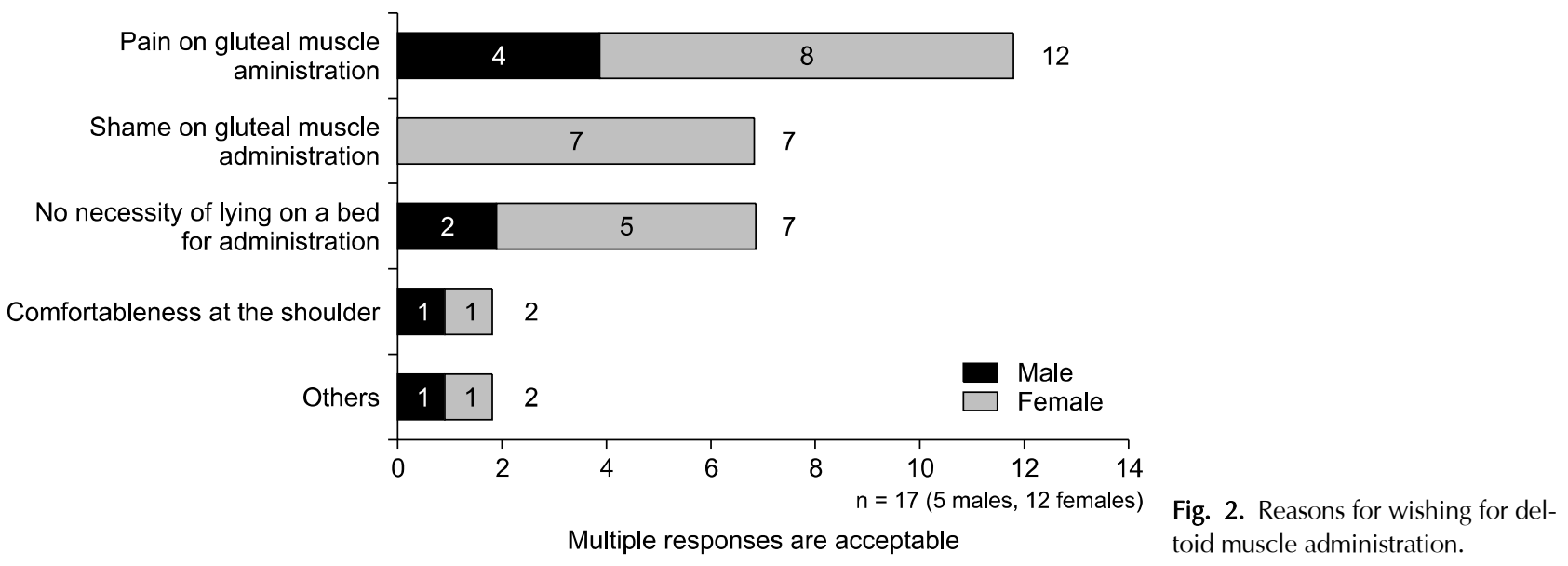



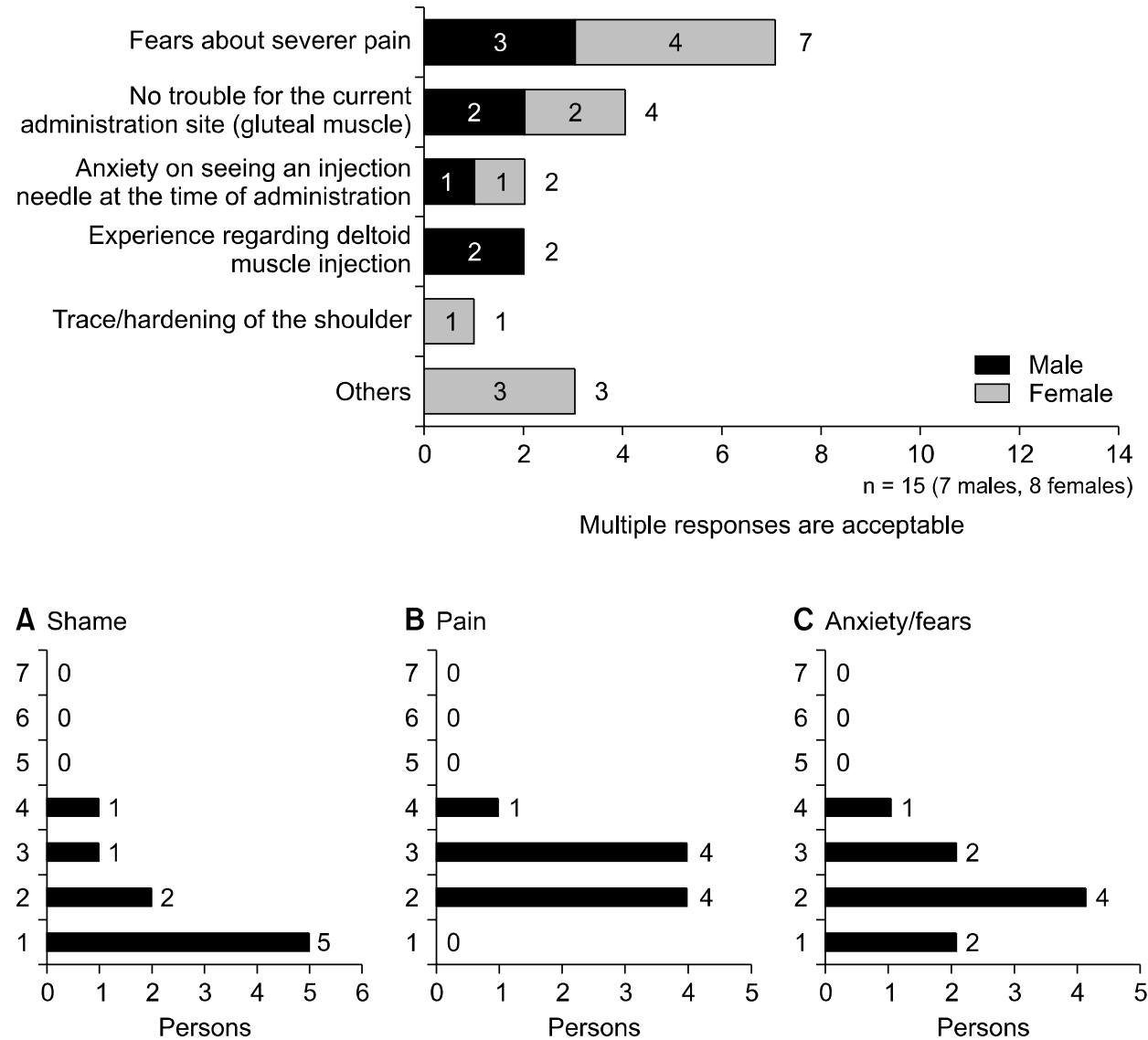
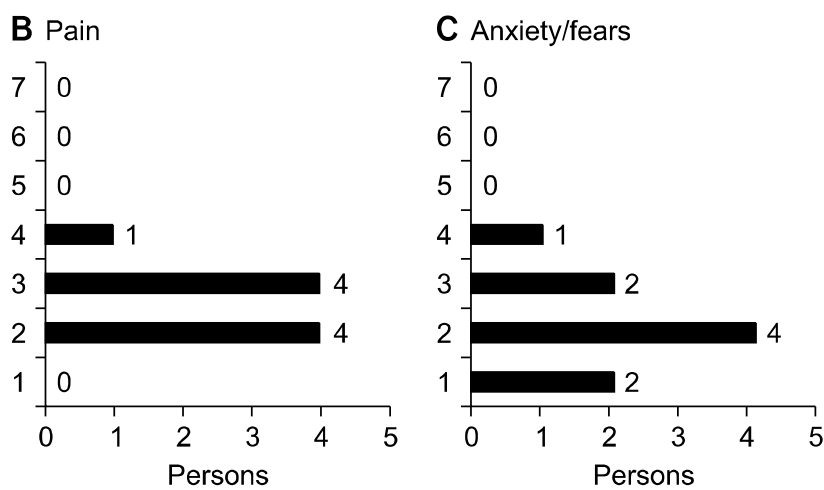

Fig. 4. Assessment of shame (A), pain (B), anxiety/fears (C) and satisfaction (D) in the deltoid muscle continuation group.

anxious/fearful”, "anxious/fearful”, or "slightly anxious/ fearful" (Fig. 4C). To the question "How about the degree of satisfaction in comparison with the previous administration site (gluteal muscle)?", 1 patient selected "relatively satisfied", 2 selected "satisfied", 5 selected "slightly satisfied", and 1 selected "no change". No patient selected "no satisfaction", "not satisfied", or "not relatively satisfied” (Fig. 4D).

\section{Survey 3: Questionnaire regarding discontinuation}

Of the 17 patients who wished for deltoid muscle administration, it was discontinued within 3 months in 7 $(41.2 \%)$, and gluteal muscle administration was again introduced. The reasons for discontinuation included "protracted pain (pain persisting for a few days after administration)" in 6 patients, "being unable to raise the arm for a while" in 1, "heavy shoulder" in 1, and "action pain" in 1 (Fig. 5).
Fig. 3. Reasons why patients did not wish for deltoid muscle administration.

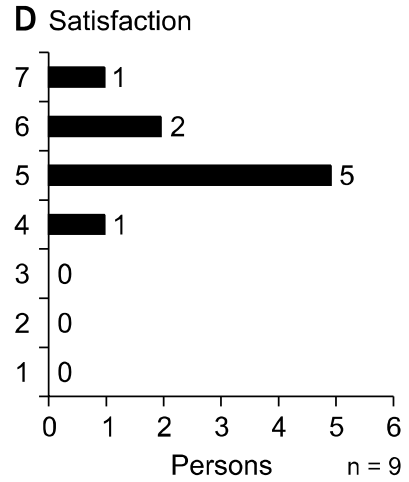

\section{Changes in the CGI Score Before and After Administration-site Switching}

We evaluated changes in the severity of psychiatric symptoms before and after administration-site switching using the CGI-S in a group in which deltoid muscle administration was continued for 3 months (deltoid muscle continuation group) and a group in which it was discontinued within 3 months, and gluteal muscle administration was again introduced (deltoid muscle discontinuation group). In the former, the CGI-S scores before administration-site switching and after 3 months were $5.0 \pm 1.9$ and $3.9 \pm 1.4$, respectively, showing a significant difference (Fig. 6). In the latter, the values were $3.7 \pm 0.5$ and $3.6 \pm 1.0$, respectively, showing no significant difference (Fig. 6).

\section{DISCUSSION}

In this study, we investigated the acceptance for the proposal to switch from gluteal to deltoid injection of ari- 


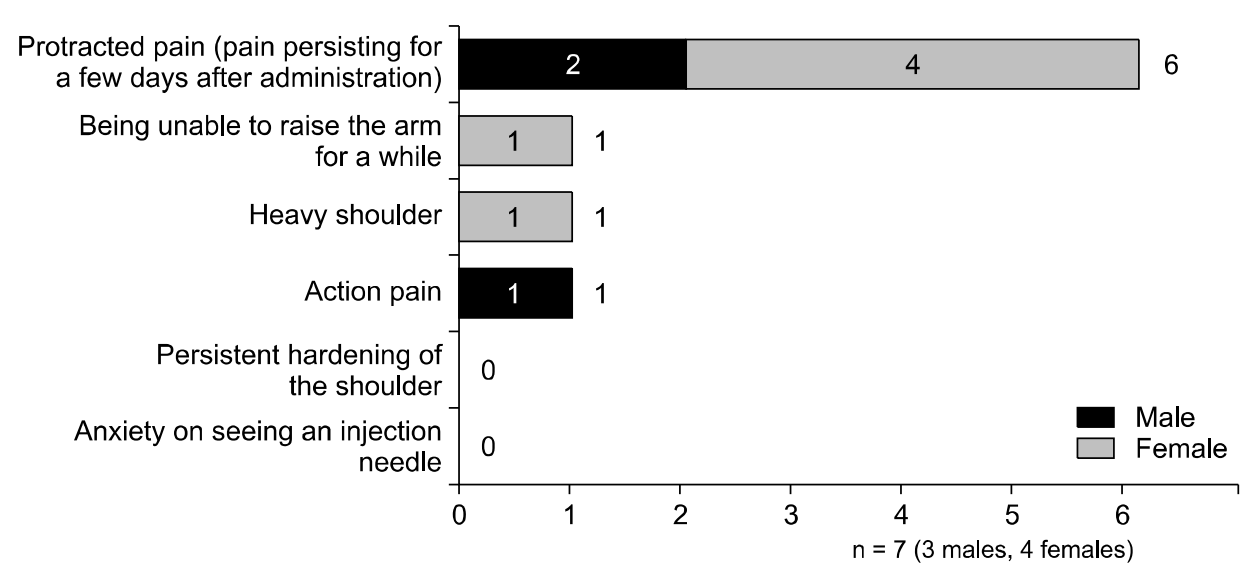

Multiple responses are acceptable
Fig. 5. Reasons for the discontinuation of deltoid muscle administration.
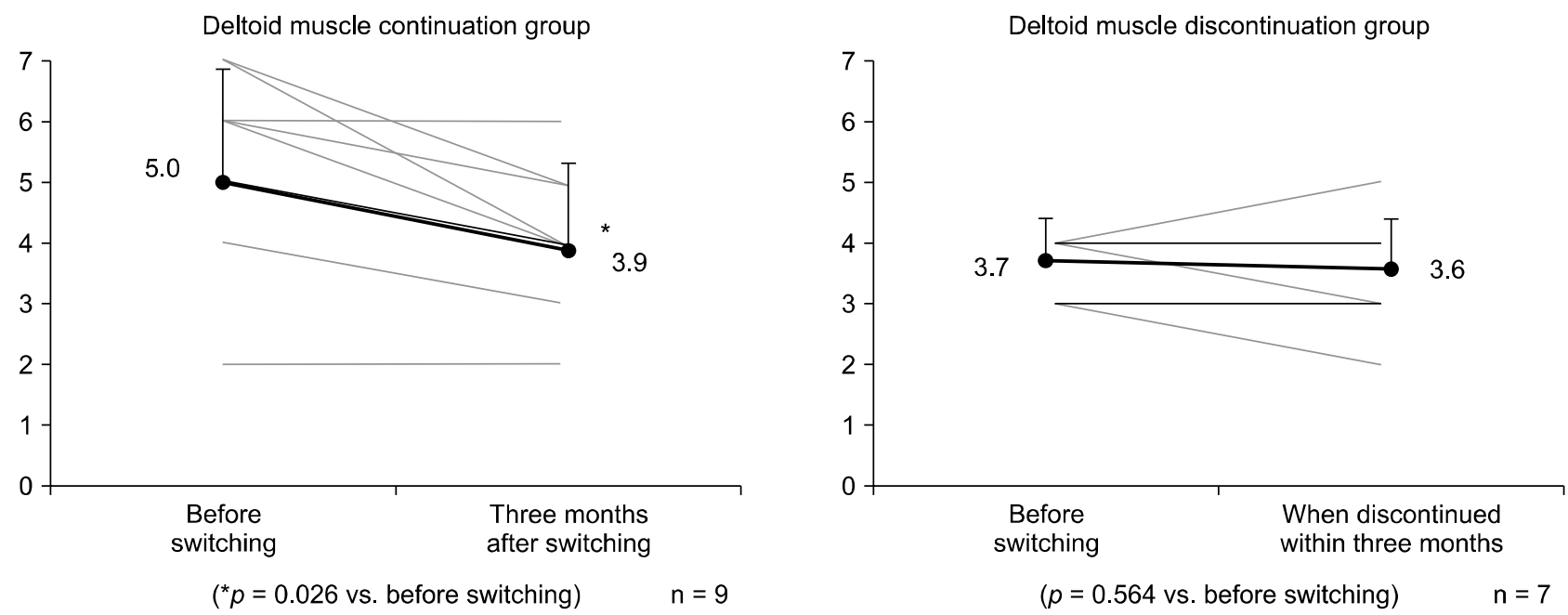

Fig. 6. Changes in Clinical Global Impressions-Severity before and after administration-site switching.

piprazole LAI in schizophrenic patients who had received aripiprazole LAI administration into the gluteal muscle for $\geq 6$ months. The results showed that $53 \%$ of the subjects wished to switch the administration site to the deltoid muscle. As the reason, 85\% selected "pain on gluteal muscle administration". On the other hand, $86 \%$ of patients who did not wish to switch the administration site to the deltoid muscle selected "deltoid muscle administration may be more painful" as the reason. Several surveys regarding $\mathrm{LAI}$ acceptance indicate that factors influencing LAI acceptance include pain and anxiety/fears about injection $[8,16,17]$. In the light of these studies, our results also showed that many patients were anxious about injection-related "pain" when introducing LAI and when selecting an injection site.

Of the subjects, 17 (53\%) wished to switch gluteal mus- cle administration to deltoid muscle administration. In 7 $(41 \%)$ of these, deltoid muscle administration was discontinued within 3 months, and gluteal muscle administration was again introduced. In most patients, the reason for the above result was severer pain at the injection site. On the other hand, an improvement in "pain" assessment in comparison with gluteal muscle administration was achieved in patients who had continuously received deltoid muscle administration for 3 months. This suggests that there are individual differences in the sense of "pain" at the administration site. Furthermore, most patients who again switched the administration site to the gluteal muscle complained of severer pain on deltoid muscle administration, indicating that patients emphasize injection-related "pain" when selecting an administration site. In this study, improvements in "pain", "shame", and "anxiety/fears" in 
comparison with gluteal muscle administration were achieved in patients who could continue deltoid muscle administration; this may have contributed to highly rated "satisfaction". Therefore, it may be useful to recommend deltoid muscle administration to patients complaining of pain on gluteal muscle administration for continuing LAI therapy.

In a previous study regarding acceptance for risperidone LAI administration into the deltoid muscle [14], $57 \%$ of patients responding to a questionnaire wished to switch to deltoid muscle administration. A survey after 3 months showed that deltoid muscle administration had been continued in $60 \%$ of patients in the switching group, whereas it was again switched to gluteal muscle administration in $40 \%$. This was consistent with the results of this study. The reasons for wishing for deltoid muscle administration included "no necessity of taking clothes off" and "no necessity of lying on a bed". The reasons why patients did not wish for deltoid muscle administration included "fears about severer pain". In a previous study regarding the administration site of paliperidone LAI [18], the reasons for wishing for deltoid muscle administration included "simple administration", "less shameful than gluteal muscle administration", and "less painful than gluteal muscle administration”. However, some patients reported that pain on deltoid muscle administration was more marked than on gluteal muscle administration [18]. The results of these previous studies support those of this study.

In addition, we examined the patient background in the deltoid muscle continuation and discontinuation groups. Considering that a primary factor for the continuation of deltoid muscle administration is the reduction of pain, and that a primary factor for its discontinuation is an increase in pain, we investigated individual differences in pain by comparing the patient background between the deltoid muscle continuation and discontinuation groups. Such differences in pain were examined with respect to the needle thickness, which may be associated with pain on injection, and arm muscle volume. The results were compared, regarding a body weight of $90 \mathrm{~kg}$ as a reference, as it was prescribed that a $22 \mathrm{G}$ needle should be used in patients weighing $\geq 90 \mathrm{~kg}$ for aripiprazole LAI administration, and that a $23 \mathrm{G}$ needle should be used in those weighing $<90 \mathrm{~kg}$ [11]. There was no significant difference between patients weighing $\geq 90 \mathrm{~kg}$ and those weighing $<90 \mathrm{~kg}$ (data not shown). In addition, we examined the results with respect to sex, as there is a gender difference in the muscle volume. However, there was no significant difference (data not shown). Thus, we could not find any factor for individual differences in pain on injection based on the results of this study.

Of the 17 patients who wished to switch to deltoid muscle administration, 7 selected "gluteal muscle administration is shameful" as the reason. All of them were female. This suggests that females particularly resist gluteal muscle administration when selecting an administration site, tending to select deltoid muscle administration. Therefore, for females, deltoid muscle administration should be recommended at the start of aripiprazole LAI therapy.

Furthermore, a significant improvement in the CGI-S score, which reflects the severity of psychiatric symptoms, was achieved in the deltoid muscle continuation group. A study regarding the pharmacokinetics and safety of aripiprazole LAI administered into the deltoid muscle indicated that there was no difference in its blood concentration between deltoid and gluteal muscle administration methods [12]. The significant improvement in the CGI-S score obtained in this study suggests that patients' mental stress for the administration site may influence psychiatric symptoms. Therefore, the selection of a better administration site for patients may reduce mental stress for injection, contributing to an improvement in adherence.

As the limitations of this study, the number of subjects was small, and it was impossible to sufficiently examine factors for the selection of an administration site or injection-related "pain". However, this study showed that pain/shame on gluteal muscle administration and inconvenience on taking clothes off/lying on a bed influenced the selection of an administration site; in particular, injection-related pain was involved in the selection of an administration site. Furthermore, females particularly considered gluteal muscle administration shameful, resisting it. Therefore, it may be necessary for health care professionals to inquire to patients of their preference for the site of injection after explaining different administration sites to them so that administration sites appropriate for individual patients may be selected. If patients complain of severe pain at a selected administration site, switching to another administration site should be considered. The selection of a more preferable admin- 
istration site for patients may reduce mental stress for injection, contributing to an improvement in LAl adherence. Deltoid muscle aripiprazole LAI administration was newly approved, increasing options for administration sites. This may provide the simplicity of administration and reduce patients' mental stress.

It didn't include the symptom severity measures except CGI-S and did not show the state of art technique such as pharmacokinetic measures, either. Both is also the serious limitation in our study. There are mainly three scales such as the Brief Psychiatric Rating Scale (BPRS) [19], the Positive and Negative Syndrome Scale (PANSS) [20], and the CGI-S [15] to measure the symptom severity of schizophrenia. In a previous study, it has been reported that the BPRS/PANSS score corresponds to the CGI-S [21]. Furthermore, because the CGI-S on relative change rather than on absolute change of symptoms [21] it may be useful in studies of short duration. To address the second issue, it has been reported that no differences occurred in the multiple-dose pharmacokinetic data were observed when patients received the first dose of aripiprazole in the deltoid site vs. the gluteal site [12]. Thus, it is possible that the improvement of symptoms after switching from gluteal administration to deltoid administration was due to the degree of mental stress regarding pain from repeated gluteal administration in the present study.

In conclusion, injection-related "pain" markedly influenced aripiprazole LAI acceptance and the choice of an injection site, and there were individual differences in the sense of pain related to administration sites. Therefore, it is necessary to select administration sites appropriate for individual patients. An increased number of options for aripiprazole $\mathrm{LAI}$ administration sites may facilitate simple administration and reduce patients' mental stress, leading to the widespread use of LAI.

\section{- Conflicts of Interest}

No potential conflict of interest relevant to this article was reported.

\section{Author Contributions}

Conceptualization: Hiroyuki Kamei, Shigeki Yamada, Nakao Iwata. Data acquisition: Yuki Homma, Ippei Takeuchi, Genta Hajitsu, Kaori Tozawa, Masakazu Hatano. Formal analysis: Yuki Homma, Aiko Fukui, Manako Hanya. Funding: Hiroyuki Kamei. Supervision:
Hiroyuki Kamei. Writing-original draft: Hiroyuki Kamei. Writing — review \& editing: Shigeki Yamada, Nakao Iwata.

\section{ORCID}

Hiroyuki Kamei

https://orcid.org/0000-0003-3778-2352

Yuki Homma

https://orcid.org/0000-0002-0247-3800

Ippei Takeuchi

Genta Hajitsu

https://orcid.org/0000-0002-7923-164X

Kaori Tozawa

https://orcid.org/0000-0002-9472-1786

Masakazu Hatano

Aiko Fukui

https://orcid.org/0000-0002-7198-8701

Manako Hanya

https://orcid.org/0000-0001-7032-878X

https://orcid.org/0000-0002-0567-5782

Shigeki Yamada

https://orcid.org/0000-0003-2255-5157

Nakao Iwata

https://orcid.org/0000-0002-3891-1323

https://orcid.org/0000-0003-3189-6076

\section{REFERENCES}

1. Weiden PJ, Kozma C, Grogg A, Locklear J. Partial compliance and risk of rehospitalization among California Medicaid patients with schizophrenia. Psychiatr Serv 2004;55:886-891.

2. Leucht S, Tardy M, Komossa K, Heres S, Kissling W, Davis JM. Maintenance treatment with antipsychotic drugs for schizophrenia. Cochrane Database Syst Rev 2012;(5):CD008016.

3. Lacro JP, Dunn LB, Dolder CR, Leckband SG, Jeste DV. Prevalence of and risk factors for medication nonadherence in patients with schizophrenia: a comprehensive review of recent literature. J Clin Psychiatry 2002;63:892-909.

4. National Collaborating Centre for Mental Health (UK). Psychosis and schizophrenia in adults: treatment and management. London: National Institute for Health and Care Excellence (UK);2014.

5. Leucht C, Heres S, Kane JM, Kissling W, Davis JM, Leucht S. Oral versus depot antipsychotic drugs for schizophrenia--a critical systematic review and meta-analysis of randomised long-term trials. Schizophr Res 2011;127:83-92.

6. Olivares JM, Rodriguez-Morales A, Diels J, Povey M, Jacobs A, Zhao Z, et al. Long-term outcomes in patients with schizophrenia treated with risperidone long-acting injection or oral antipsychotics in Spain: results from the electronic Schizophrenia Treatment Adherence Registry (e-STAR). Eur Psychiatry 2009; 24:287-296.

7. Llorca PM, Bobes J, Fleischhacker WW, Heres S, Moore N, Bent-Ennakhil N, et al. Baseline results from the European non-interventional Antipsychotic Long acTing injection in schizOphrenia (ALTO) study. Eur Psychiatry 2018;52:85-94.

8. Jaeger M, Rossler W. Attitudes towards long-acting depot antipsychotics: a survey of patients, relatives and psychiatrists. Psychiatry Res 2010;175:58-62.

9. Kane JM, Sanchez R, Zhao J, Duca AR, Johnson BR, McQuade $\mathrm{RD}$, et al. Hospitalisation rates in patients switched from oral anti-psychotics to aripiprazole once-monthly for the management of schizophrenia. J Med Econ 2013;16:917-925. 
10. Potkin SG, Preda A. Aripiprazole once-monthly long-acting injectable for the treatment of schizophrenia. Expert Opin Pharmacother 2016;17:395-407.

11. Otsuka Pharmaceutical Co., Ltd. The manual of ABILIFY $Y^{R}$ (aripiprazole). Tokyo:Otsuka Pharmaceutical Co., Ltd.;2016.

12. Raoufinia A, Peters-Strickland T, Nylander AG, Baker RA, Eramo A, Jin N, et al. Aripiprazole once-monthly $400 \mathrm{mg}$ : comparison of pharmacokinetics, tolerability, and safety of deltoid versus g/uteal administration. Int J Neuropsychopharmacol 2017;20:295-304.

13. Yin J, Collier AC, Barr AM, Honer WG, Procyshyn RM. Paliperidone palmitate long-acting injectable given intramuscularly in the deltoid versus the g/uteal muscle: are they therapeutically equivalent? J Clin Psychopharmacol 2015;35: 447-449.

14. Heres S, Frobose T, Hamann J, Leucht S, Maino K, Reichhart $\mathrm{T}$, et al. Patients' acceptance of the deltoid application of risperidone long-acting injection. Eur Neuropsychopharmacol 2012;22:897-901.

15. Busner J, Targum SD. The clinical global impressions scale: applying a research tool in clinical practice. Psychiatry (Edgmont)
2007:4:28-37.

16. Geerts P, Martinez G, Schreiner A. Attitudes towards the administration of long-acting antipsychotics: a survey of physicians and nurses. BMC Psychiatry 2013;13:58.

17. Sugawara N, Kudo S, Ishioka M, Sato Y, Kubo K, YasuiFurukori N. Attitudes toward long-acting injectable antipsychotics among patients with schizophrenia in Japan. Neuropsychiatr Dis Treat 2019;15:205-211.

18. Hough D, Lindenmayer JP, Gopal S, Melkote R, Lim P, Herben $\mathrm{V}$, et al. Safety and tolerability of deltoid and g/uteal injections of paliperidone palmitate in schizophrenia. Prog Neuropsychopharmacol Biol Psychiatry 2009;33:1022-1031.

19. Overall JE, Gorham DR. The brief psychiatric rating scale. Psychol Rep 1962;10:790-812.

20. Kay SR, Fiszbein A, Opler LA. The positive and negative syndrome scale (PANSS) for schizophrenia. Schizophr Bull 1987; 13:261-276.

21. Leucht S, Kane JM, Etschel E, Kissling W, Hamann J, Engel RR. Linking the PANSS, BPRS, and CGI: clinical implications. Neuropsychopharmacology 2006;31:2318-2325. 